\section{Bitte lächeln, Frau Schmidt!}

\author{
Die Budgetierung beutelt uns Ärzte in allen Bereichen enorm. Wirt- \\ schaftlichkeit wird gepredigt - zu viele und teure Untersuchungen \\ würden durchgeführt - eine sparsamste Medikamentenverordnung \\ wird angemahnt!
}

$\mathrm{N}$ un hat das Bundessozialgericht entschieden, dass Vertragsärzte Leistungen selbst dann anbieten müssen, auch wenn die Kosten nicht gedeckt sind. Dazu sollen alle typischen zum Gebietsstandard gehörenden Leistungen zählen. Die Pflicht zur Leistungserbringung erstreckt sich nach dem Urteil der Kasseler Richter auf die Kernleistungen des jeweiligen Fachgebietes. Und hier beißt sich der Hund in den Schwanz.

\section{Was sind typische Leistungen im Gebiet und was Kernleistungen?}

Oder sind Kernleistungen automatisch auch typische Leistungen? Gehören ein oraler Provokationstest, eine Arzneimitteltestung und/oder eine Testung mit Hymenopterengiften oder Naturlatexproteinen zu den Kernleistungen der allergologischen Diagnostik? Nach meinen Erfahrungen und den mir zugeleiteten Überweisungen auch von Allergologen gehören diese Untersuchungen wohl nicht zu den Kernleistungen unseres Bereiches.

Dieser so genannte "Gebietsstandard“ ist bisher nicht definiert. Wenn sich nicht die ärztlichen Gremien und insbesondere auch die Fachverbände dieser Fragestellung annehmen, werden es die Gerichte übernehmen, wobei dann wie schon häufig für den einzelnen Arzt nicht viel Gutes zu erwarten ist.

Aufgrund dieser Lücke relativiert sich der Kasseler Richterspruch. Kernleistungen wie allergologische Anamnesen und Beratungen, Hauttests, organbezogene Provokationstests und serologische Untersuchungsmethoden werden von der großen Mehrheit der Aller- gologen angeboten, so dass es dem Einzelnen auch weiterhin überlassen bleibt, ob er darüber hinaus andere Leistungen unter Berücksichtigung seiner persönlichen wirtschaftlichen Lage anbieten kann oder will.

Bei der Leistungserbringung werden wir genötigt, notfalls auch unwirtschaftlich zu handeln. Bei der Medikamentenverordnung ist es genau entgegengesetzt: Hier werden äußerste Sparsamkeit und Ausschöpfung aller Sparmaßnahmen gefordert.

Der Arzt wird somit zum Zwitter mit richterlich verordnetem schizophrenen Wirtschaftlichkeitsdenken. Man gewinnt den Eindruck, je höher instanzlich die richterlichen Entscheidungen gefällt werden, desto realitätsfremder werden sie. Ich habe versucht in meinem Bekanntenkreis Personen in kaufmännischen Berufen unsere Situation zu schildern. In der Mehrzahl der Fälle bekam ich die Antwort: „Hör auf zu scherzen!"

Eine Dekadenzkurve verläuft zunächst langsam abflachend, knickt dann, um schließlich immer steiler abzufallen. Sind wir schon am Knickpunkt angelangt?

— Ärzteverbände und auch die KBV sind um Änderungen bemüht. Diese in die Tat umzusetzen ist mühsam, was jeder Verbandsfunktionär weiß.

- Die Bundesministerin für Gesundheit, Frau Ulla Schmidt, soll die Probleme bis zur nächsten Legislaturperiode „über- bzw. weglächeln“.

\section{Termine}

\section{Frankfurt, 17. November 2001}

Tagung der Regionalgruppe Hessen

\section{Thema:}

— Sinusitis - Pathogenese, Diagnostik und Therapie (Pilze, Bakterien, Allergie)

Organisation: Dr. M. Schiffmann, Prof. Dr. W. W. Schlenter HNO-Abteilung St. Marienkrankenhaus Richard-Wagner-Straße 14 D-60318 Frankfurt Tel.: 069 / $1563-266$ Fax: $069 / 1563-140$

\section{Dessau, 1. Dezember 2001}

Tagung der Regionalgruppe Sachsen-Anhalt

Thema:

— Allergologie interdisziplinär Aktuelle Trends in der Diagnostik und Therapie

\section{Organisation:}

Prof. Dr. D. Lübbe

Klinik und Poliklinik für

Hautkrankheiten

Kromeyerstraße 5-6

D-06097 Halle

Tel.: 0345 / 5573 - 911

Fax: $0345 / 5573-941$

Es bleibt zu hoffen, dass Patienten und deren Organisationen in Zusammenarbeit mit den Ärzten - notfalls auch mit Protestreaktionen - die Politik zu einem schnelleren Handeln bewegen können.

Dr. W. Kersten, Moers

Impressum „Der ÄDA informiert"

Verantwortlich für den Inhalt: Prof. Dr. Ludger Klimek

Redaktion: Dr. Corinna K. Leitnaker

Urban \& Vogel Medien und Medizin Verlagsgesellschaft mbH \& Co. KG, Neumarkter Straße 43 81637 München 